\title{
Breast cancer metastasis and the lymphatic system (Review)
}

\author{
MUNAZZAH RAHMAN $^{1}$ and SULMA MOHAMMED ${ }^{1-3}$ \\ ${ }^{1}$ Department of Comparative Pathobiology, Purdue University; ${ }^{2}$ Purdue Center for Cancer Research; \\ ${ }^{3}$ Bindley Bioscience, Purdue Discovery Park, West Lafayette, IN 47907, USA
}

Received July 22, 2014; Accepted February 23, 2015

DOI: $10.3892 / 01.2015 .3486$

\begin{abstract}
Breast cancer remains the leading cause of cancer mortality worldwide, despite a significant decline in death rates due to early detection. The majority of cancer mortalities are due to the metastasis of tumor cells to other organs. Metastasis or tumor cell dissemination occurs via the hematogenous and lymphatic systems. For many carcinomas, the dissemination of tumor cells via lymphatic drainage of the tumor is the most common metastatic route. Such lymphatic drainage collects at the regional lymph nodes and the dissection and pathological examination of these nodes for lodged cancer cells is the gold standard procedure to detect metastasis. The present report provides an overview of the lymphatic system and its clinical significance as a prognostic factor, in addition to the interactions between the primary tumor and its microenvironment, and the influence of genomic subtypes on the resulting organ-specific pattern of tumor cell dissemination. It also examines the seemingly protracted asymptomatic period, during which the disseminated cells remain dormant, leading to the manifestation of metastasis decades after the successful treatment of the primary tumor.
\end{abstract}

\section{Contents}

1. Introduction

2. The lymphatic system and lymph formation

3. The lymphatic system and metastasis

4. Lymphangiogenesis and lymphatic metastasis

5. Clinical significance of the lymphatic system

6. Conclusion

Correspondence to: Dr Sulma Mohammed, Department of Comparative Pathobiology, Purdue University, 725 Harrison Street, West Lafayette, IN 47907, USA

E-mail: mohammes@purdue.edu

Key words: lymph, lymphatic system, metastasis, breast cancer, lymphangiogenesis

\section{Introduction}

Breast cancer is the most commonly diagnosed malignancy worldwide, with an estimated 1.7 million cases diagnosed in 2012 (1). The incidence rate for the USA in 2013 alone was 232,340 cases, accounting for $29 \%$ of all new cancer cases among females (2). Early diagnosis via mammographic screening, and implementation of post-surgical systemic adjuvant therapy has led to a significant decrease in breast cancer mortalities in developed countries. However, breast cancer remains the leading cause of cancer death despite these advances, with $\sim 90 \%$ of these mortalities being due to metastasis of tumor cells to other organs. The median survival rate of females with metastatic disease is only 2-3 years (3). One of the most critical steps in identifying metastasis and staging a newly diagnosed patient with breast cancer is determining whether the cancer has spread to the regional lymph nodes. Regional lymph nodes are the primary sites of lymphatic drainage from all areas of the breast, and the extent of their involvement in breast cancer is considered to be a strong predictor of recurrences and survival. Therefore, the lymphatic system is important in metastasis. This study reviews the lymphatic system and the cellular composition of the lymph fluid, and discusses their relevance in breast cancer metastasis and as sources for therapeutic targets.

\section{The lymphatic system and lymph formation}

The lymphatic and blood circulatory systems complement one another in the maintenance of body tissue homeostasis. The lymphatic system regulates tissue fluid balance and facilitates interstitial protein transport. Extravasated plasma and proteins from blood capillaries are collected in the interstitial space, forming the lymph fluid, and returned to the blood circulation. Therefore, the biochemical composition and color of lymph varies depending on the location and permeability of the lymphatic vessel involved. In general, the protein composition of the lymph is comparable to that of interstitial fluid, and is less concentrated than blood plasma (4). The lymphatic system may be divided into five sections based on size and functionality: The lymphatic capillaries, lymphatic collecting vessels, lymph nodes, lymphatic trunks and ducts.

The lymphatic capillaries drain the net exudate and facilitate convective protein transport through the interstitium. They possess irregular and wider lumen when compared with vascular capillaries, and are closely associated with the 
adjacent interstitial areas. These blind-ended structures are comprised of a single layer of endothelial cells and a discontinuous basement membrane of $10-50 \mu \mathrm{m}$ in diameter (5). The luminal and abluminal surfaces of these cells have invaginations and cytoplasmic vesicles. The extensive superimposition of endothelial cells forms clefts of interendothelial junctions that act as valves, aiding in the transendothelial transport of molecules of 10-25 $\mathrm{nm}$ in diameter into the lumen. Tight junctions and adherens junctions are infrequent, and represent focal points of adhesion. Anchoring filaments of fine stranded fibrillin tether endothelial cells to the extracellular matrix (ECM). An increase in interstitial fluid volume causes the sensitive anchoring filament to exert tension on the lymphatic capillaries, thereby opening the intercellular junctions and increasing the luminal volume. As fluid enters the lumen and decreases the pressure difference across the vessel wall, the junctions begin to close, preventing retrograde flow into the interstitium. While the majority of the fluids are reabsorbed into post-capillary venules, a small net fluid flux into the tissues occurs due to the hydrostatic and osmotic pressure gradients created by the extravasated proteins. The lymphatic system and ECM maintain the fluid equilibrium. The composition and organization of the ECM determines the elasticity and hydration of the tissues. Therefore, damage to the ECM leads to lymphatic dysfunction due to the alterations of the interstitium (6).

The lymph subsequently flows into lymphatic collecting vessels that are $>200 \mu \mathrm{m}$ in diameter and are not tethered to the ECM. The vessels are composed of three layers of cells, with a similar composition to arterial walls (7). The adventitia is comprised of fibroblasts, connective tissue and nerves. The media is formed from smooth muscle that supports the circumferential hoop stress, intermixed with collagen and elastic fibers that are lined by an intimal layer of endothelial cells. Retrograde flow of lymph is prevented via unidirectional valves, and the segments of collecting lymphatics between the valves are termed lymphangions. Lymphangions serve as contractile compartments that work in conjunction with the valves to facilitate propulsion of lymph, at $\sim 10 \mu \mathrm{m} / \mathrm{sec}$, into the subsequent lymphangion compartment against a pressure gradient. They contract cyclically at a rate of 1-15 times/minute, a result of the intrinsic wall motion generated via the stimulus of smooth muscle cells and the compression caused by arterial pulsations and skeletal muscle contraction. However, lymphangions are also prone to regulation via prostaglandins and thromboxane. The lymphatic collecting vessels are classified as prenodal (afferent) or postnodal (efferent) lymphatics to denote the direction of lymph flow via the lymph nodes.

Lymph nodes are surrounded by a thick capsule and are compartmentalized into narrow fluid crevices via connective tissue trabeculae (8). The structure is organized into the cortex, medulla and sinuses. The cortex is further differentiated into the periphery, containing lymphatic nodules composed of B-lymphocytes that are concentrated in the primary follicles and germinal centers, and the deep cortex, which contains the T-lymphocytes. Specialized postcapillary venules termed high endothelial venules (HEVs) are localized to the cortex. These HEVs express ligands that direct the flow of lymphocytes from blood into the appropriate part of the lymph node. The medulla contains cords comprised of plasma cells. Lymph sinuses containing lymphocytes and macrophages form the inner core of the lymph node. Lymph, along with lymphocytes, antigen processing macrophages and dendritic cells, flows into the subcapsular sinus of the lymph nodes via the afferent lymphatic collecting vessels. Afferent lymphatic vessels predominate, resulting in the substantial deceleration of lymph flow, thereby allowing the aforementioned macrophages and lymphocytes to cleanse and filter lymph of foreign debris and bacteria (9). Hence, lymph nodes are important components of the immune system and are considered to be the secondary center of the immune response. The lymph subsequently exits the lymph node at its hilus via the efferent lymphatic collecting vessels. The drained materials are routed to efferent vessels, where they exit the lymph node and proceed through lymphatic circulation to main collecting ducts, the lymphatic trunks, and finally anastomose with the vascular system via the junction of the left jugular and subclavian veins.

\section{The lymphatic system and metastasis}

The inherent characteristics of the lymphatic physiology serve as the primary route for tumor cell metastasis. The increasing size of the tumor triggers a rise in the intratumoral interstitial fluid pressure, and interstitial fluid is released as the system attempts to achieve homeostasis. Unlike the vascular vessels, the lymphatic vessels are highly permeable; the flow rate is approximately 100-500x slower, and coupled with lesser shearing stresses due to vasodilation. Therefore, the lymphatic route is superior in facilitating tumor cell dissemination (10). Distinguishing between lymphatic endothelial and systemic endothelial cells via immunohistochemical staining has allowed studies to confirm tumor cell dispersion via afferent lymphatics and lymphangiogenesis, and implicates the lymphatics as the most significant metastatic route (11).

Metastasis is a complex and inefficient process, which requires sequential regulation of a number of biological steps prior to the presentation of overt disease. This involves tumor cell dissociation, angiogenesis of the primary tumor, intravasation, survival and diffusion through the systemic and lymphatic circulation, adhesion to organ walls, extravasation, establishment of metastatic foci at the organ parenchyma, and finally, manifestation of clinically apparent metastasis via the generation of secondary tumors (12). Although mechanisms such as angiogenesis occur during normal physiological conditions, the stringent regulation that typically leads to the cessation of the processes malfunctions during malignant invasion, thereby allowing superfluous events to persist despite the detrimental outcome. Hematogenous dissemination is considered to be the predominant avenue for metastasis, however, this is dependent upon the origin of the primary tumor, and it is possible that the circulating tumor cells may favor the lymphogenic and transcoelomic routes. Breast tumors, in particular, preferentially utilize the lymphatic system for tumor cell dissemination.

A salient aspect of metastasis is the potential of many tumor types to preferentially colonize various organs. Breast cancer cells possess an organ-specific pattern of dissemination and metastasize to bone (47-60\%) (13), and subsequently to the liver (19-20\%) (14), lung (16-34\%) (15), and brain (10-16\%) (16). The tumor cells migrate from the primary tumor to the sentinel lymph node. However, no direct 
lymphatic routes to the preferred target organs have been identified, therefore the tumor cells eventually exit via the efferent lymphatic vessels and utilize the venous system or the nascent blood vessels that serve lymph nodes to merge with the systemic circulation. While this was previously considered to be a passive process (17), studies have revealed that chemokine receptors $\mathrm{C}-\mathrm{X}-\mathrm{C}$ chemokine receptor type 4 and $\mathrm{C}-\mathrm{C}$ chemokine receptor type 7 are overexpressed in organs that are primary targets for metastasis (liver, bone and lungs), and may promote migration of their ligands, $\mathrm{C}-\mathrm{X}-\mathrm{C}$ motif chemokine 12/stromal cell-derived factor $1 \alpha$ and chemokine (C-C Motif) ligand 21 (18). Additionally, studies have suggested that the primary tumor secretes vascular endothelial growth factor (VEGF) A and other soluble factors to define a pre-metastatic niche in the target organs, thereby demarcating where the tumor cells should preferentially metastasize (19).

However, studies have indicated that, while patients exhibiting metastasis appear to have an elevated relapse risk, this does not ensure recurrent disease (20). Paget (21) explains this phenomenon with the suggestion of the 'seed and soil' hypothesis, emphasizing the interactions of the tumor cells and the microenvironment of the target organ for the establishment of metastasis. Furthermore, while metastasis to distant organs is a rapid process in other cancers, breast cancer metastasis may manifest decades after the successful treatment of the primary tumor. Studies that have modeled dormancy mathematically have revealed that the protracted asymptomatic period possesses intermittent growth punctuated with periods of quiescence (22). This indicates that the tumor cells and the target organ microenvironment evolve to attain malignancy. These tumor cells may exist as solitary dormant cells, pre-angiogenic metastasis and vascularized metastasis, simultaneously. Solitary dormant cells undergo reversible quiescence by employing redundant mechanisms to avoid apoptosis, including activation of the Hedgehog pathway, dysregulation of transforming grow th factor- $\beta$ and other anti-apoptotic proteins including B-cell lymphoma 2 (23). These cells are assisted in quiescence via interactions with the target organ ECM, and involvement of urokinase-type plasminogen activator receptor, epidermal growth factor receptor, extracellular signal-regulated kinases and p38 regulation $(24,25)$. This state of dormancy renders immunity to these cells, as chemotherapy agents target rapidly proliferating cells. Preangiogenic metastasis involves avascular tumors that exhibit high proliferation rates, which are offset by elevated apoptosis. However, inhibition of the potent angiogenesis inhibitor, thrombospondin, allows this avascular metastasis to undergo drastic alterations and progress to a highly vascularized and rapidly proliferating tumor (26).

Organ-specific metastasis, tumor dormancy and recurrence were further influenced by the disease biology. Recently, new understanding of this biology using cDNA microarrays has revealed that breast cancer is heterogeneous and comprised of distinct entities with different metastatic patterns, which lead to varying prognoses. These molecular entities may be termed basal-like, luminal-A, luminal-B, and human epidermal growth factor receptor 2 (HER2)-positive $(27,28)$. The basal-like tumors are significantly associated with a poor prognosis as they are characterized by triple-negative hormone receptors [i.e., estrogen receptor (ER) negative, progesterone receptor (PR) negative, and HER2 negative].
These tumors preferentially metastasize to the brain, lung and distant lymph nodes, in addition to liver and bone, albeit at a significantly lower rate (29). Investigations that examined metastasis to axillary lymph nodes resulted in equivocal conclusions. Furthermore, basal-like subtypes tend to recur sooner, have a negative prognosis and a significantly shorter survival following metastasis. The luminal subtype is the most frequently diagnosed and is further divided into luminal-A and luminal-B on the basis of their characteristics. Luminal-A tumors tend to be hormone receptor-positive and demonstrate low proliferative activity. They are associated with the lowest risk of lymph node metastasis and locoregional recurrence (30). Luminal-B tumors are predominantly ER-positive tumors and exhibit highly proliferative activity. Luminal-B tumors also tend to have poorer prognosis compared with luminal-A tumors, and are associated with the highest locoregional recurrence rate. The two luminal subtypes preferentially metastasize to bone (31). HER2-positive subtypes are represented by tumors with high expression of the ERBB2 gene and tend to be poorly differentiated (27), of a higher grade and more likely to involve axillary lymph nodes. HER2-positive tumors are associated with a significantly higher rate of brain, liver and lung metastases. While HER 2 subtypes tend to exhibit aggressive phenotypes and present with the worst metastasis survival rates (32), they are amenable to targeted therapy.

\section{Lymphangiogenesis and lymphatic metastasis}

While the promoting effect of angiogenesis and vascularization of the tumor in the progression of the disease is well documented, there is little information with regard to lymphangiogenesis and its function in metastasis. Certain studies have indicated that the tumors are devoid of lymphatic vessels, while others have suggested that tumors invade and destroy lymphatic vessels (33). Furthermore, other studies have indicated that tumor cells may induce lymphangiogenesis, some form of lymphatic sprouting, or hyperplasia in close proximity to the periphery of the tumors (17). Therefore, the pertinent question is whether lymphangiogenesis is necessary for lymphatic metastasis. Although it is possible for lymphatic metastasis to occur via preexisting vessels that were incorporated into the tumors, there is evidence to suggest that increased lymphatic vessel density due to lymphangiogenesis significantly improves metastasis (34).

The utilization of lymphangiogenesis promoting factors as markers has facilitated the examination of tumors to determine the existence of an intratumoral lymphatic network. For instance, VEGF-C has previously been demonstrated to stimulate the growth of lymphatic vascular endothelium, which has been observed to be overexpressed in breast cancer cells. Stimulation by VEGF-A and C has also been implicated in lymphangiogenesis and correlated with sentinel node metastasis (35). Animal xenograph studies utilized lymphatic vessel endothelial hyaluronan receptor (LYVE-1), a marker of lymphatic endothelium, to demonstrate that VEGF-C potently increased intratumoral lymphangiogenesis. The study concluded that lymphangiogenesis was positively and strongly correlated to lymph node and lung metastasis (36). Elevated levels of the receptor tyrosine kinase Tie-2 and its ligands, 
angiopoietin-1 and angiopoietin-2, have also been implicated in facilitating lymphatic metastasis (37).

The formation of an intratumoral lymphatic network may promote metastatic tumor spread by creating increased opportunities for metastatic tumor cells to exit the primary tumor site, whether fully functional in fluid transport or not. Recent studies have implicated aggressive tumors in furthering lymphangiogenesis and thereby facilitating the metastatic process (38). Tumor invasiveness has been shown to be associated with the expression of the VEGF angiogenic growth factor family, particularly VEGF-C and VEGF-D. An upregulation of its cognate receptor tyrosine kinase, VEGF receptor 3 (VEGFR3), has been detected in peripheral cancer cells, as well as in blood vessel endothelium. The VEGFR3 receptor is generally located on the lymphatic endothelium and is therefore considered to be a viable marker for intratumoral lymphatic networks. Overexpression of this receptor has been implicated in lymph node metastasis and a negative prognosis (39). However, it lacks lymphatic vessel specificity in human cancer, as tumor blood vessels may also express VEGFR3.

Nevertheless, studies have directly implicated VEGF-C and VEGF-D in lymphatic metastasis $(40,41)$. Animal studies have demonstrated that tumors overexpressing these proteins have higher rates of tumor lymphangiogenesis and metastasis. It was concluded that the overexpression of VEGF-C was positively correlated with tumor incidence; however, it did not appear to affect tumor volume, angiogenesis or transition from adenoma to carcinoma (42). VEGF-C may promote metastasis by increasing the number of lymphatic vessels in the vicinity of tumor cells, thereby facilitating the dissemination of tumor cells from the primary tumor site $(43,44)$. The growth rate of the tumor, angiogenesis and the formation of metastases appear to be promoted by VEGF-D (45). However, other studies conducted to investigate the association of VEGF-C and VEGF-D expression with metastasis have resulted in contradictory conclusions $(46,47)$. These ambiguous results may be attributed to tumor microenvironment variations, including degrees of proteolytic processing, dissimilarities in tumor tissues, and interactions with other lymphangiogenic factors.

\section{Clinical significance of the lymphatic system}

Tumor size and axillary lymph node status are independent measures of prognosis (48). However, it is an established fact that tumor size is directly proportional to the number of axillary lymph nodes affected by metastasis (49). The 5-year survival rates improved markedly from 45.5 to $96.3 \%$ when tumor size decreased from 5 to $2 \mathrm{~cm}$ (48).

Lymph nodes act as a reservoir for tumor cells to take root and form metastatic tumors. Therefore, the extent of lymph node involvement and regional lymph node status is considered to be crucial in patient prognosis (50). The dissection and assessment of the axillary lymph nodes (ALND) is an essential and routine component in the process of staging and determination of adjuvant therapy modalities. The National Surgical Adjuvant Bowel and Breast Project B-04 trial randomized patients to radical mastectomy (including the removal of breast tissue, axillary lymph nodes and pectoralis muscles), total mastectomy (with axillary lymph nodes and pectoralis muscles left intact) or total mastectomy with axillary radiation. The two cohorts that did not receive ALND had an $18.6 \%$ increased risk of developing axillary lymph node metastasis compared with the cohort that received radical mastectomy treatment (51). Additionally, studies have revealed that ALND reduces axillary node metastases recurrence risk by $1-3 \%$ (52). However, ALND may result in significant postoperative morbidity $(60 \%)$ and severe complications, including lymphedema of the arm, seroma, sensory numbness, paresthesia, pain and infection (53). Lymphedema is of particular concern, as it occurs in $6-30 \%$ of patients, and no curative therapy is available (54). Furthermore, with the advent of improved screening modalities, breast cancer is now being diagnosed and treated at an early stage, prior to lymph node metastasis. However, as ALND is an established procedure (55), it is conducted irrelevant of metastatic status, potentially jeopardizing patient health unnecessarily $(56,57)$.

These issues compelled the investigation of procedures capable of precisely staging disease progression, and inducing fewer side effects. Studies examining the potential of the sentinel node biopsy (SNB) indicated that it may be an effective alternative for ALND (58). Radioactive technetium (Tc99m), isosulfan or methylene blue is injected into the breast, and the first one to four lymph nodes that absorb the dye are removed and evaluated. The probability of tumor cell dissemination to the axillary nodes is $<10 \%$ if the SNB analysis is negative. SNB accurately determines the axillary nodal status, is cost effective and is correlated with a shorter postoperative time; however, the sensitivity of the procedure is inconsistent, and it is associated with a false negative rate average of $8.5 \%$ across 69 studies including 10,454 patients (59).

Imaging techniques such as positron emission tomography (PET), magnetic resonance imaging (MRI) and computed tomography (CT) perfusion have also been investigated with regard to their ability to determine axillary node metastasis. PET utilizes a radiolabeled glucose analogue that allows the visualization of abnormal metabolic features associated with malignancy, based on the enhanced glycolytic rate, and therefore heightened tracer uptake, of malignant tissues compared with surrounding benign tissues (60). CT utilizes ionizing radiation as the basis of image contrast and provides anatomical imaging. MRI relies on the magnetic resonance characteristics of hydrogen nuclei to distinguish between soft tissues and pathologies, thereby providing information regarding size and morphology. A number of studies have used these techniques in conjunction to improve accuracy. PET combined with CT allows for precise anatomical localization of abnormalities (61). However, PET combined with MRI, while associated with fewer adverse events, led to a lower sensitivity and specificity than SNB (62). Furthermore, the clinical significance of these modalities is yet to be established.

Fluorescent labeling of LYVE-1, a lymphatic endothelial cell receptor that lines lymphatic vessels, is the most widely used technique for the assessment of tumor cell migration via the lymphatic system (63). However, in vivo application of this process is prone to cytotoxicity, immune responses, photobleaching, blinking or strong light scattering, and background auto-fluorescence. The advent of nanotechnology has introduced an alternate fluorescence technique that labels 
disseminated tumor cells. However, while nanomaterial, such as quantum dots, allow for long-term tracking, they have the propensity to undergo endocytosis by lymph node macrophages and therefore are not useful for depicting lymphatic drainage (64). When utilized simultaneously, these techniques may provide valuable information.

The significance of lymphangiogenesis and its contribution towards metastasis has become more apparent over recent decades due to the identification of lymphatic specific biomarkers that are absent or expressed at relatively low levels in vascular endothelium (65). These include podoplanin, LYVE-1, prospero homeobox protein 1 (PROX1), $\beta$-chemokine receptor D6 (66) and macrophage mannose receptor (67). Podoplanin, a glomerular podocyte membrane mucoprotein, is expressed solely on lymphatic capillary endothelium (68). LYVE-1 is a CD44 homolog that is involved in hyaluronan homeostasis or in the regulation of cellular trafficking to the lymph nodes (69). PROX1 is a homeobox transcription factor that induces lymphatic, lineage-specific differentiation and that is essential for the embryonic development of the lymphatic system from the blood vascular system (70).

In 2007, gene expression profiling-based assays, including Oncotype DX, Prosigna (PAM50), MammaPrint and Mammostrat, were used to measure gene activity in primary tumor cells (71). Oncotype DX utilizes four genomic markers (ER, PR, HER2 and Ki-67) to generate predictions and classify patients according to recurrence risk (72). Prosigna (PAM50) utilizes 50 classifier genes and five control genes to identify the five intrinsic subtypes of breast cancer (73). MammaPrint utilizes a 70-gene panel and is the first FDA-approved, commercialized microarray-based multigene assay designed to individualize treatment (74). Mammostrat uses an index of five independent markers in conjunction with histopathological information to provide insight into cell proliferation and the hormone receptor status of a tumor (75). While these assays include the status of the lymph node in their recurrence score, they only assess the early metastatic potential of the tumors, and have not demonstrated improved outcomes.

\section{Conclusion}

The purpose of this investigation was to provide understanding of breast cancer metastasis and the underlying mechanisms of the lymphatic system in facilitating the progression of the disease. It provided an overview of the lymphatic system, the interactions between the primary tumor and the microenvironment as well as the influence of genomic subtypes on the resulting organ-specific pattern of tumor cell dissemination. The seemingly protracted asymptomatic period during which the disseminated cells remain dormant, leading to the manifestation of metastasis decades after the successful treatment of the primary tumor was also discussed as well as the clinical significance of the lymphatic system as a prognostic factor and the biomarkers that have been discovered thus far.

Characterization of the lymphatic system is essential in order to provide insight into significant predictive associations with metastatic risk. A combination of the currently identified biomarkers may be utilized to elucidate associations between lymphatic vessel density, lymphatic metastasis to distant organs and the resultant prognosis of breast cancer.

\section{References}

1. Torre LA, Bray F, Siegel RL, Ferlay J, Lortet-Tieulent J and Jemal A: Global cancer statistics, 2012. CA Cancer J Clin 65: 87-108, 2015

2. Siegel R, Naishadham D and Jemal A: Cancer statistics, 2013. CA Cancer J Clin 63: 11-30, 2013.

3. Liotta LA and Kohn EC: The microenvironment of the tumour-host interface. Nature 411: 375-379, 2001.

4. Tanner GA: Kidney function. In: Medical Physiology: Principles for Clinical Medicine. Rhoades RA and Bell DR (eds). 3rd edition. Lippincott Williams \& Wilkins, Philadelphia, p420, 2009.

5. Alitalo K, Tammela T and Petrova TV: Lymphangiogenesis in development and human disease. Nature 438: 946-953, 2005.

6. Rossi A, Weber E, Sacchi G, Maestrini D, Di Cintio F and Gerli R: Mechanotransduction in lymphatic endothelial cells. Lymphology 40: 102-113, 2007.

7. Scavelli C, Weber E, Aglianò M, Cirulli T, Nico B, Vacca A and Ribatti D: Lymphatics at the crossroads of angiogenesis and lymphangiogenesis. J Anat 204: 433-449, 2004.

8. Young B, Lowe JS, Stevens A, Heath JW and Deakin PJ (eds): Immune systems. In: Wheater's Functional Histology: A Text and Colour Atlas. 5th edition. Churchill Livingstone, New York, NY, pp219-220, 2006

9. von Andrian UH and Mempel TR: Homing and cellular traffic in lymph nodes. Nat Rev Immunol 3: 867-878, 2003.

10. Fischer M, Franzeck UK, Herrig I, Costanzo U, Wen S, Schiesser M, Hoffmann U and Bollinger A: Flow velocity of single lymphatic capillaries in human skin. Am J Physiol 270: H358-H363, 1996.

11. Mohammed RA, Martin SG, Gill MS, Green AR, Paish EC and Ellis IO: Improved methods of detection of lymphovascular invasion demonstrate that it is the predominant method of vascular invasion in breast cancer and has important clinical consequences. Am J Surg Pathol 31: 1825-1833, 2007.

12. Chambers AF, Groom AC and MacDonald IC: Dissemination and growth of cancer cells in metastatic sites. Nat Rev Cancer 2: 563-572, 2002.

13. Coleman RE and Rubens RD: The clinical course of bone metastases from breast cancer. Br J Cancer 55: 61-66, 1987.

14. Selzner M, Morse MA, Vredenburgh JJ, Meyers WC and Clavien PA: Liver metastases from breast cancer: Long-term survival after curative resection. Surgery 127: 383-389, 2000.

15. Minn AJ, Gupta GP, Siegel PM, Bos PD, Shu W, Giri DD, Viale A, Olshen AB, Gerald WL and Massagué J: Genes that mediate breast cancer metastasis to lung. Nature 436: 518-524, 2005.

16. Lin NU, Bellon JR and Winer EP: CNS metastases in breast cancer. J Clin Oncol 22: 3608-3617, 2004.

17. Hartveit E: Attenuated cells in breast stroma: The missing lymphatic system of the breast. Histopathology 16: 533-543, 1990.

18. Fernandis AZ, Prasad A, Band H, Klösel R and Ganju RK: Regulation of CXCR4-mediated chemotaxis and chemoinvasion of breast cancer cells. Oncogene 23: 157-167, 2004.

19. Psaila B and Lyden D: The metastatic niche: Adapting the foreign soil. Nat Rev Cancer 9: 285-293, 2009.

20. Klein CA: The systemic progression of human cancer: A focus on the individual disseminated cancer cell - the unit of selection. Adv Cancer Res 89: 35-67, 2003.

21. Paget S: The distribution of secondary growths in cancer of the breast. Lancet 133: 571-573, 1889.

22. Demicheli R: Tumour dormancy: Findings and hypotheses from clinical research on breast cancer. Semin Cancer Biol 11: 297-306, 2001

23. Liu S, Dontu G, Mantle ID, Patel S, Ahn NS, Jackson KW, Suri $\mathrm{P}$ and Wicha MS: Hedgehog signaling and Bmi-1 regulate self-renewal of normal and malignant human mammary stem cells. Cancer Res 66: 6063-6071, 2006.

24. Ranganathan AC, Adam AP, Zhang L and Aguirre-Ghiso JA: Tumor cell dormancy induced by p38SAPK and ER-stress signaling: An adaptive advantage for metastatic cells? Cancer Biol Ther 5: 729-735, 2006.

25. Noh H, Hong S and Huang S: Role of urokinase receptor in tumor progression and development. Theranostics 3: 487-495, 2013.

26. Jones T, Neboori H, Wu H, Yang Q, Haffty BG, Evans S, Higgins S and Moran MS: Are breast cancer subtypes prognostic for nodal involvement and associated with clinicopathologic features at presentation in early-stage breast cancer? Ann Surg Oncol 20: 2866-2872, 2013. 
27. Watnick RS, Cheng YN, Rangarajan A, Ince TA and Weinberg RA: Ras modulates Myc activity to repress thrombospondin-1 expression and increase tumor angiogenesis. Cancer Cell 3: 219-231, 2003.

28. Perou CM, Sørlie T, Eisen MB, et al: Molecular portraits of human breast tumours. Nature 406: 747-752, 2000.

29. Rakha EA, El-Rehim DA, Paish C, Green AR, Lee AH, Robertson JF, Blamey RW, Macmillan D and Ellis IO: Basal phenotype identifies a poor prognostic subgroup of breast cancer of clinical importance. Eur J Cancer 42: 3149-3156, 2006.

30. Kennecke H, Yerushalmi R, Woods R, Cheang MC, Voduc D, Speers CH, Nielsen TO and Gelmon K: Metastatic behavior of breast cancer subtypes. J Clin Oncol 28: 3271-3277, 2010.

31. Howland NK, Driver TD, Sedrak MP, Wen X, Dong W, Hatch S, Eltorky MA and Chao C: Lymph node involvement in immunohistochemistry-based molecular classifications of breast cancer. J Surg Res 185: 697-703, 2013.

32. Smid M, Wang Y, Klijn JG, Sieuwerts AM, Zhang Y, Atkins D, Martens JW and Foekens JA: Genes associated with breast cancer metastatic to bone. J Clin Oncol 24: 2261-2267, 2006.

33. Carmeliet P and Jain RK: Angiogenesis in cancer and other diseases. Nature 407: 249-257, 2000.

34. Colleoni M, Rotmensz N, Maisonneuve P, et al: Prognostic role of the extent of peritumoral vascular invasion in operable breast cancer. Ann Oncol 18: 1632-1640, 2007.

35. Hirakawa S, Brown LF, Kodama S, Paavonen K, Alitalo K and Detmar M: VEGF-C-induced lymphangiogenesis in sentine lymph nodes promotes tumor metastasis to distant sites. Blood 109: 1010-1017, 2007.

36. Mattila MM, Ruohola JK, Karpanen T, Jackson DG, Alitalo K and Härkönen PL: VEGF-C induced lymphangiogenesis is associated with lymph node metastasis in orthotopic MCF-7 tumors. Int J Cancer 98: 946-951, 2002.

37. Caine GJ, Stonelake PS, Lip GY and Blann AD: Changes in plasma vascular endothelial growth factor, angiopoietins, and their receptors following surgery for breast cancer. Cancer Lett 248: 131-136, 2007.

38. Oliver $\mathrm{G}$ and Detmar M: The rediscovery of the lymphatic system: Old and new insights into the development and biological function of the lymphatic vasculature. Genes Dev 16: 773-783, 2002.

39. Nakamura Y, Yasuoka H, Tsujimoto M, Yang Q, Imabun S, Nakahara M, Nakao K, Nakamura M, Mori I and Kakudo K: Flt-4-positive vessel density correlates with vascular endothelial growth factor-d expression, nodal status, and prognosis in breast cancer. Clin Cancer Res 9: 5313-5317, 2003.

40. Nakamura Y, Yasuoka H, Tsujimoto M, Imabun S, Nakahara M, Nakao K, Nakamura M, Mori I and Kakudo K: Lymph vessel density correlates with nodal status, VEGF-C expression, and prognosis in breast cancer. Breast Cancer Res Treat 91: $125-132,2005$.

41. Nakamura Y, Yasuoka H, Tsujimoto M, Yang Q, Imabun S, Nakahara M, Nakao K, Nakamura M, Mori I and Kakudo K: Prognostic significance of vascular endothelial growth factor D in breast carcinoma with long-term follow-up. Clin Cancer Res 9: 716-721, 2003.

42. Mandriota SJ, Jussila L, Jeltsch M, et al: Vascular endothelial growth factor-C-mediated lymphangiogenesis promotes tumour metastasis. EMBO J 20: 672-682, 2001.

43. Van Trappen PO and Pepper MS: Lymphangiogenesis and lymph node microdissemination. Gynecol Oncol 82: 1-3, 2001.

44. Witte MH, Way DL, Witte CL and Bernas M: Lymphangiogenesis: Mechanisms, significance and clinical implications. EXS 79: 65-112, 1997

45. Wigle JT, Harvey N, Detmar M, et al: An essential role for Prox1 in the induction of the lymphatic endothelial cell phenotype. EMBO J 21: 1505-1513, 2002

46. Watanabe O, Kinoshita J, Shimizu T, Imamura H, Hirano A, Okabe T, Aiba M and Ogawa K: Expression of a CD44 variant and VEGF-C and the implications for lymphatic metastasis and long-term prognosis of human breast cancer. J Exp Clin Cancer Res 24: 75-82, 2005.

47. Currie MJ, Hanrahan V, Gunningham SP, Morrin HR, Frampton C, Han C, Robinson BA and Fox SB: Expression of vascular endothelial growth factor $\mathrm{D}$ is associated with hypoxia inducible factor (HIF-1alpha) and the HIF-1alpha target gene DEC1, but not lymph node metastasis in primary human breast carcinomas. J Clin Pathol 57: 829-834, 2004.

48. Carter CL, Allen C and Henson DE: Relation of tumor size, lymph node status, and survival in 24,740 breast cancer cases. Cancer 63: 181-187, 1989.
49. Ioachim HL and Medeiros LJ (eds): Metastatic breast carcinoma. In: Ioachim's Lymph Node Pathology. 4th Edition. Lippincott Williams \& Wilkins, Philadelphia, p606, 2009.

50. Woodward WA, Strom EA, Tucker SL, et al: Changes in the 2003 American Joint Committee on Cancer staging for breast cancer dramatically affect stage-specific survival. J Clin Oncol 21: 3244-3248, 2003

51. Fisher B, Jeong JH, Anderson S, Bryant J, Fisher ER and Wolmark N: Twenty-five-year follow-up of a randomized trial comparing radical mastectomy, total mastectomy, and total mastectomy followed by irradiation. N Engl J Med 347: $567-575,2002$.

52. Veronesi U, Viale G, Paganelli G, et al: Sentinel lymph node biopsy in breast cancer: Ten-year results of a randomized controlled study. Ann Surg 251: 595-600, 2010

53. Warmuth MA, Bowen G, Prosnitz LR, Chu L, Broadwater G, Peterson B, Leight G and Winer EP: Complications of axillary lymph node dissection for carcinoma of the breast: A report based on a patient survey. Cancer 83: 1362-1368, 1998

54. Petrek JA and Heelan MC: Incidence of breast carcinoma-related lymphedema. Cancer 83: 2776-2781, 1998.

55. Goldhirsch A, Glick JH, Gelber RD and Senn HJ: Meeting highlights: International Consensus Panel on the Treatment of Primary Breast Cancer. J Natl Cancer Inst 90: 1601-1608, 1998.

56. Robinson DS, Senofsky GM and Ketcham AS: Role and extent of lymphadenectomy for early breast cancer. Semin Surg Oncol 8: 78-82, 1992.

57. Fleissig A, Fallowfield LJ, Langridge CI, et al: Post-operative arm morbidity and quality of life. Results of the ALMANAC randomised trial comparing sentinel node biopsy with standard axillary treatment in the management of patients with early breast cancer. Breast Cancer Res Treat 95: 279-293, 2006.

58. Krag DN, Anderson SJ, Julian TB, et al: Sentinel-lymph-node resection compared with conventional axillary-lymph-node dissection in clinically node-negative patients with breast cancer: Overall survival findings from the NSABP B-32 randomised phase 3 trial. Lancet Oncol 11: 927-933, 2010.

59. Lyman GH, Giuliano AE, Somerfield MR, et al; American Society of Clinical Oncology: American Society of Clinical Oncology guideline recommendations for sentinel lymph node biopsy in early-stage breast cancer. J Clin Oncol 23: 7703-7720, 2005.

60. Strauss LG and Conti PS: The applications of PET in clinical oncology. J Nucl Med 32: 623-648, 1991.

61. Eubank WB, Mankoff DA, Schmiedl UP, Winter TC III, Fisher ER, Olshen AB, Graham MM and Eary JF: Imaging of oncologic patients: Benefit of combined CT and FDG PET in the diagnosis of malignancy. AJR Am J Roentgenol 171: $1103-1110,1998$

62. Cooper KL, Meng Y, Harnan S, Ward SE, Fitzgerald P, Papaioannou D, Wyld L, Ingram C, Wilkinson ID and Lorenz E: Positron emission tomography (PET) and magnetic resonance imaging (MRI) for the assessment of axillary lymph node metastases in early breast cancer: Systematic review and economic evaluation. Health Technol Assess 15: iii-iv, 1-134, 2011.

63. McElroy M, Hayashi K, Garmy-Susini B, Kaushal S, Varner JA, Moossa AR, Hoffman RM and Bouvet M: Fluorescent LYVE-1 antibody to image dynamically lymphatic trafficking of cancer cells in vivo. J Surg Res 151: 68-73, 2009.

64. Hama Y, Koyama Y, Urano Y, Choyke PL and Kobayashi H: Simultaneous two-color spectral fluorescence lymphangiography with near infrared quantum dots to map two lymphatic flows from the breast and the upper extremity. Breast Cancer Res Treat 103: 23-28, 2007.

65. Stacker SA, Achen MG, Jussila L, Baldwin ME and Alitalo K: Lymphangiogenesis and cancer metastasis. Nat Rev Cancer 2: 573-583, 2002 .

66. Nibbs RJ, Kriehuber E, Ponath PD, et al: The beta-chemokine receptor D6 is expressed by lymphatic endothelium and a subset of vascular tumors. Am J Pathol 158: 867-877, 2001.

67. Irjala H, Johansson EL, Grenman R, Alanen K, Salmi M and Jalkanen S: Mannose receptor is a novel ligand for L-selectin and mediates lymphocyte binding to lymphatic endothelium. J Exp Med 194: 1033-1042, 2001

68. Schacht V, Ramirez MI, Hong YK, et al: T1alpha/podoplanin deficiency disrupts normal lymphatic vasculature formation and causes lymphedema. EMBO J 22: 3546-3556, 2003.

69. Jackson DG: Biology of the lymphatic marker LYVE-1 and applications in research into lymphatic trafficking and lymphangiogenesis. APMIS 112: 526-538, 2004. 
70. Hong YK, Harvey N, Noh YH, Schacht V, Hirakawa S, Detmar M and Oliver G: Prox1 is a master control gene in the program specifying lymphatic endothelial cell fate. Dev Dyn 225: 351-357, 2002

71. Marchionni L, Wilson RF, Marinopoulos SS, Wolff AC, Parmigiani G, Bass EB and Goodman SN: Impact of gene expression profiling tests on breast cancer outcomes. Evid Rep Technol Assess (Full Rep) 160: 1-105, 2007.

72. Paik S, Shak S, Tang G, et al: A multigene assay to predict recurrence of tamoxifen-treated, node-negative breast cancer. N Engl J Med 351: 2817-2826, 2004.
73. Nielsen TO, Parker JS, Leung S, et al: A comparison of PAM50 intrinsic subtyping with immunohistochemistry and clinical prognostic factors in tamoxifen-treated estrogen receptor-positive breast cancer. Clin Cancer Res 16: 5222-5232, 2010

74. Cuadros $\mathrm{M}$ and Llanos A: Validation and clinical application of MammaPrint ${ }^{\circledR}$ in patients with breast cancer. Med Clin (Barc) 136: 627-632, 2011 (In Spanish).

75. Bartlett JM, Thomas J, Ross DT, et al: Mammostrat as a tool to stratify breast cancer patients at risk of recurrence during endocrine therapy. Breast Cancer Res 12: R47, 2010. 